\title{
Fuzzy Velocity and Fuzzy Acceleration in Fractional Order Motion
}

\author{
A. Ates, B.B. Alagoz, G.T. Alisoy, C Yeroglu and H.Z. Alisoy
}

\begin{abstract}
Fractional calculus presents a new perspective for solution of scientific and engineering problems. There are still many fields where fractional calculus promise fresh understanding of real world problems. In this study, we present an investigation on fractional order motion, where we extend our comprehension from velocity and acceleration concepts to a fuzzy velocity and acceleration domains. Here, fundamentally, we suggest a debate on interpretation of fractional-order derivative system on the bases of integer-order derivative knowledge. We observed that the continuous fractional-order motion equation set can be considered to cover the discrete integer-order motion equation set and physical meaning of fractional-order motion systems can be better understood by using a fuzzy conceptualization of integer-order derivative systems according to the dissimilarity metric.
\end{abstract}

Index Terms - Fractional-order derivative models, physical interpretation of fractional-order models, fractional-order motion analysis

\section{INTRODUCTION}

$\mathrm{T}$ HERE has been renewed interest to fractional-order system due to its practical outcomes in engineering and applied science [1-14]. The first discussion on a fractional-order derivative (FOD) can be traced back to 1690s when L'Hospital mentioned to Leibniz about what if ' $n$ ' order of a derivative becomes a none-integer [15]. Since then, a question of how a non-integer orders, namely a fractional-order, derivative can be meaning in a physical sense. Although FOD were attracted attention by Euler and Lagrange, systematical labors were started at the beginning and middle of the 19th century by Liouville (1832), Holmgren (1864), Riemann (1953) [1, 3]. In practical sense, some approximation definitions for calculation of fractional-order integrations and derivations were given by Grünwald-Letnikov, Riemann -Liouville and Caputo [1-6].

A. Ates is with the Computer Engineering Department, Inonu University, Malatya, Turkey, (e-mail: abdullah.ates@inonu.edu.tr).

B.B. Alagozis with the Electrical and Electronics Engineering Department, Inonu University, Malatya, Turkey (e-mail: baykant.alagoz@inonu.edu.tr).

G.T. Alisoy is with the Mathematics Department, Namik Kemal University, Tekirdag, Turkey, (e-mail: galisoy@nku.edu.tr).

C. Yeroglu is with the Computer Engineering Department, Inonu University, Malatya, Turkey, (e-mail: c.yeroglu@inonu.edu.tr).

H.Z. Alisoy is with the Electrionics and Comminication Engineering Department, Namik Kemal University, Tekirdag, Turkey, (e-mail: halisoy@nku.edu.tr).

Copyright (C) BAJECE
There were several works aiming physical interpretation of fractional derivatives: Machado presented a probabilistic interpretation of the fractional-order differentiation using Grünwald-Letnikov definition [16].

Podlubny explained a fractional derivative as the projection of the area below a function over a nonlinear time scale with a deformation parameter $\alpha$ [17]. Moshrefi-Torbati et. al. considered the Riemman-Lioville fractional derivative of a function as the convolution of this function with a kernel $\left(\mathrm{h}_{\infty}\right)$ [18]. Molz et. al. demonstrated that a fractional derivative can be identified as a typical superposition integral over the spatial domain of the Levy diffusion process [19]. Giannantoni present extensive [20] work on physical meaning in linear differential equations of fractional order. He stated that "a time differential problem described by one fractional differential equation generates new 'special' functions (the 'binary', 'ternary', 'quaternary' functions and so on) which can be interpreted as being the mathematical description of the evolution of a unique system, made up of a prefixed number of parts, which are in turn so strictly related to each other that they form one sole entity [20]. J. Sabatier et. al. indicate that neither Riemann-Liouville nor Caputo definitions for fractional differentiation can be used to take into account initial conditions in a convenient way from a physical point of view via a counter-example [21].

R. E. Gutierrez et. al. present a relationship between concepts like position and speed of an object according to GrünwaldLetnikov and Reiman-Liouville definition [22].

In our study, observable states of system models such as displacement, velocity, acceleration are considered as related states (parts) of FOD modeling space. Interaction between these concepts is classified by defining a distance metric, which forms fuzzy domains of observable states of physical systems.

Human conscious is shaped by observable universe. This causes a tendency to conceptualize our physical world by means of observable states, namely measurable parameters, preferably based on simplified mathematical expression. Hence, observable states are conceptualized on the base of integer-order derivatives (IOD). Is this just a coincidence or a conscious preference? The answer may be it is because of simplicity in calculation of IODs compared to FODs. However, we know that this choice has confined modeling affords of physical world into IODs modeling set. When researchers began to expand their view to include FODs last century, they came across an infinite set of a modeling space, where the behavior of real dynamical systems could be described by more compact expressions [14]. Despite the fact that it works superior in many applications as a mathematical tool, physical interpretations of FOD models are somewhat problematic. A noteworthy way to deal with these complications may be found by defining fuzzy conceptual 
domains in FOD modeling space on the base of well-known IOD modeling concepts.

This paper presents a discussion on fuzzy conceptual domains in order to enhance our understanding of FOD modeling. This can expand our view on the physical interpretation of FOD models. The continuous FOD modeling set is divided a class of fuzzy domains of conceptualized IOD modeling by means of dissimilarity metrics. Then, a FOD model can be classified into one of these fuzzy domains of IOD models that already have a physical comprehension or a physical meaning such as heat, pressure, velocity, force etc. In this fashion, we give a basic illustration on a FOD motion modeling of position data in a polynomial character. A fuzzy position, a fuzzy velocity and a fuzzy acceleration modeling domains are formed and we illustrate that any FOD model derived from the position data can be classified one of these fuzzy domains according to similarity of their characteristics. This allows identifying a FOD model of motion by associating them to a most likely physical concept.

\section{METHODOLOGY}

\section{A. Fractional-Order Calculus Basics}

Fractional integro-differential calculations are performed by $D^{\alpha}$ operator, where $\alpha$ denote the fractional order for $\alpha \in R$. When $\alpha>0, D^{\alpha}$ operator performs fractional derivation of a function. When $\alpha<0, D^{\alpha}$ operator performs fractional integration of a function. In the case of $\alpha=0$, it yields the function itself. Three most common approximate definitions were developed for $D^{\alpha}$ operator and used in calculation of fractional-order systems, which are Grünwald-Letnikov (GL), Riemann-Liouville (RL), Caputo (C) definitions [1-6].

In this study, we used $D^{\alpha}$ for $\alpha>0$ as a FOD operator. For the sake of simplicity of our explanations, we used polynomial functions with constant coefficients to model a motion of mechanical system in our example application. However, similar studies can be extended for more complex functions. Let express a constant coefficient polynomial function family as,

$$
f_{n}=\sum_{i=0}^{n} a_{i} t^{i}
$$

where $a_{i}$ are constant coefficient of the polynomials and $n$ is the degree of polynomials. A fractional-derivative of a member of this polynomial family can be expressed as,

$$
D^{\alpha} f_{n}=\sum_{i=0}^{n} a_{i} \frac{\Gamma(i+1)}{\Gamma(i-\alpha+1)} t^{i-\alpha}
$$

where $\Gamma($.$) represents gamma function.$

\section{B. Basic Definitions of A Fractional-Order Derivate Modeling Set and Application for a Constant Coefficient Polynomial Family}

Let us denote a model function of a physical event by $f$ and assume that the function $f$ was obtained from an experimental observation of physical system. A fractional-derivative modeling set of $f$ is defined as $\psi^{\alpha}=D^{\alpha} f$ for $\alpha \geq 0$ and $\alpha \in R$ . Accordingly, IOD modeling set can be defined as $\psi^{m}=D^{m} f$ for $m \geq 0$ and $m \in Z$. One can clearly see that an IOD modeling set of $f$ is utterly contained by a FOD modeling set of $f$ due to $m \in \alpha$.

A short-time average of absolute difference distance metric (STADD) to indicate the dissimilarities between elements of a fractional-derivative modeling set of $f$ is defined as follows,

$$
E_{w}^{u}=\frac{1}{t} \int_{0}^{t}\left|\psi^{u}-\psi^{w}\right| d t
$$

where $E_{w}^{u}$ for $u, w \in \alpha$, represents distance between fractional models $\psi^{u}$ and $\psi^{w}$ of a given $f$ at a time $t$. In the case of $t \rightarrow \infty$ , one obtains all-time average absolute difference distance between models with order $u$ and $w$. When a constant coefficient polynomial function family of time $t$, denoted by $f_{n}(t)$, is considered, the distance metric between fractional model $\psi^{u}$ and $\psi^{w}$ can be expressed as,

$$
E_{w}^{u}=\frac{1}{t} \int_{0}^{t}\left|\left(\frac{t^{w}-t^{u}}{t^{w u}}\right) \sum_{i=0}^{n} a_{i} \frac{\Gamma(i+1)}{\Gamma(i-\alpha+1)} t^{i}\right| d t
$$

By considering equation (2), one can reorganize equation (4) as,

$$
E_{w}^{u}=\frac{1}{t} \int_{0}^{t}\left|\left(\frac{t^{w}-t^{u}}{t^{w u}}\right) \psi^{0}\right| d t=\frac{1}{t} \int_{0}^{t}\left|t^{-u} f_{n}(t)-t^{-w} f_{n}(t)\right| d t
$$

Equation (5) implies us that fractional-derivative models of $f_{n}$ are discriminated from each other with nonlinear weighting terms, $t^{-u}$ and $t^{-w}$ in term of $E_{w}^{u}$ metric. This causes a half-orders models $(\alpha=m / 2)$ not to be in equal distance to its neighbor two IOD models. For instance, $E_{1 / 2}^{0} \neq E_{1 / 2}^{1}$. This results in an imbalanced sharing of a FOD modeling set (Imbalanced allocation) by fuzzy domains of concept models.

Now, let us diverge a fractional-order of derivative modeling of $f_{n}(t)$ as small as possible. In other words, assuming that $u=u \pm \varepsilon$ and $\varepsilon \rightarrow 0$, equation (5) can be treated as

$$
\lim _{\varepsilon \rightarrow 0} E_{w}^{u}=\lim _{\varepsilon \rightarrow 0} \frac{1}{t} \int_{0}^{t}\left|t^{-w} f_{n}(t)\left(1-t^{ \pm \varepsilon}\right)\right| d t
$$

Equation (6) tell us that near-order models $(|\mathrm{u}-\mathrm{w}|<\varepsilon)$ are more similar than those of far-order models of $f_{n}$. This property 
results in a fuzzy similarity of models. These findings are clearly demonstrated on motion modeling in further sections. When specific order models are chosen as base models or concept models, FOD modeling set can be divided into domains of concept models according to $E_{w}^{u}$ metrics. These domains are called as fuzzy domains of concepts models. Let assume $w_{1}, w_{2}$, $w_{3},,, w_{k}$, derivative order of concept models (observable states) of a physical system. A given $\alpha$ fractional-order derivate of a function belongs to a $p^{\text {th }}$ fuzzy domain of FOD modeling set, if only if there exists one minimum distance metrics to $w_{p}$-ordered concept model such that $\min \left\{E_{w_{1}}^{\alpha}, E_{w_{2}}^{\alpha}, E_{w_{2}}^{\alpha},,, E_{w_{k}}^{\alpha}\right\}=E_{w_{p}}^{\alpha}$. When there are more than one minimum metrics to concept models such that $\min \left\{E_{w_{1}}^{\alpha}, E_{w_{2}}^{\alpha}, E_{w_{2}}^{\alpha},,, E_{w_{k}}^{\alpha}\right\}=\left\{E_{w_{p 1}}^{\alpha}, E_{w_{p 2}}^{\alpha},, E_{w_{p h}}^{\alpha}\right\}, \alpha$ FOD model is said to be a boundary models of fuzzy domains of, $w_{\mathrm{p} 1}, w_{\mathrm{p} 2}$, $w_{\mathrm{p} 3},, w_{\mathrm{ph}}$ orders. In other words, $\alpha$ FOD model accommodates at the boundaries of fuzzy domains of $w_{\mathrm{p} 1}, w_{\mathrm{p} 2}, w_{\mathrm{p} 3},, w_{\mathrm{ph}}$ due to having equal distances to $w_{\mathrm{p} 1}, w_{\mathrm{p} 2}, w_{\mathrm{p} 3},, w_{\mathrm{ph}}$ order derivative models.

\section{Fuzzy Domains Interpretation of a Fractional-Order Motion Modeling Set}

In this section, we give a brief illustration on a motion equation model. For this proposes, physical concept models (Position, Velocity, Acceleration) as given in Table I are expanded a fuzzy position, a fuzzy velocity and a fuzzy acceleration domains as represented in Fig.1.

TABLE I. PHYSICAL CONCEPTS OF MOTION ANALYSIS.

\begin{tabular}{c|l|l|l}
\hline $\begin{array}{l}\text { Order of } \\
\text { Derivative }(\alpha)\end{array}$ & $\begin{array}{l}\text { Physical Concept } \\
\text { Models }\end{array}$ & $\begin{array}{l}\text { Derivative } \\
\text { Modeling }\end{array}$ & Fuzzy Domains \\
\hline 0 & Displacement & $y=f_{n}(t)$ & Fuzzy position \\
\hline 1 & Velocity & $v=D^{1} f_{n}(t)$ & Fuzzy velocity \\
\hline 2 & Acceleration & $a=D^{2} f_{n}(t)$ & Fuzzy Acceleration \\
\hline
\end{tabular}

Therefore $\alpha$ is a real number, motion model set in Fig.1(a) are continuous. Metrics of these fuzzy modeling domains can be written according to equation (3) as follows,

$$
\begin{aligned}
& P_{\alpha}=E_{0}^{\alpha}=\frac{1}{t} \int_{0}^{t}\left|\psi^{\alpha}-\psi^{0}\right| d t \\
& V_{\alpha}=E_{1}^{\alpha}=\frac{1}{t} \int_{0}^{t}\left|\psi^{\alpha}-\psi^{1}\right| d t \\
& A_{\alpha}=E_{2}^{\alpha}=\frac{1}{t} \int_{0}^{t}\left|\psi^{\alpha}-\psi^{2}\right| d t
\end{aligned}
$$

where $P_{\alpha}$ is the distance to position concept model (integer order with $\alpha=0), V_{a}$ is the distance to velocity concept model (integer order with $\alpha=1$ ) and $A_{\alpha}$ is the distance to acceleration concept model (integer order with $\alpha=2$ ). A $\psi^{\alpha}$ FOD model belongs to a fuzzy model domain $(F D)$ with a minimum distance as follows:

$$
F D=\left\{\begin{array}{cl}
\text { position domain } & \min \left\{P_{\alpha}, V_{\alpha}, A_{\alpha}\right\}=P_{\alpha} \\
\text { velocity domain } & \min \left\{P_{\alpha}, V_{\alpha}, A_{\alpha}\right\}=V_{\alpha} \\
\text { accelaration domain } & \min \left\{P_{\alpha}, V_{\alpha}, A_{\alpha}\right\}=A_{\alpha}
\end{array}\right.
$$

Equation (10) classifies a fractional-order motion equation into three fuzzy concept domains, which are a fuzzy position domain, a fuzzy velocity domain and a fuzzy acceleration domain, according to distance metrics defined by equation (7), (8) and (9). Some boundaries of these fuzzy concept domains are defined by boundary of fuzzy domains (BFD) as,

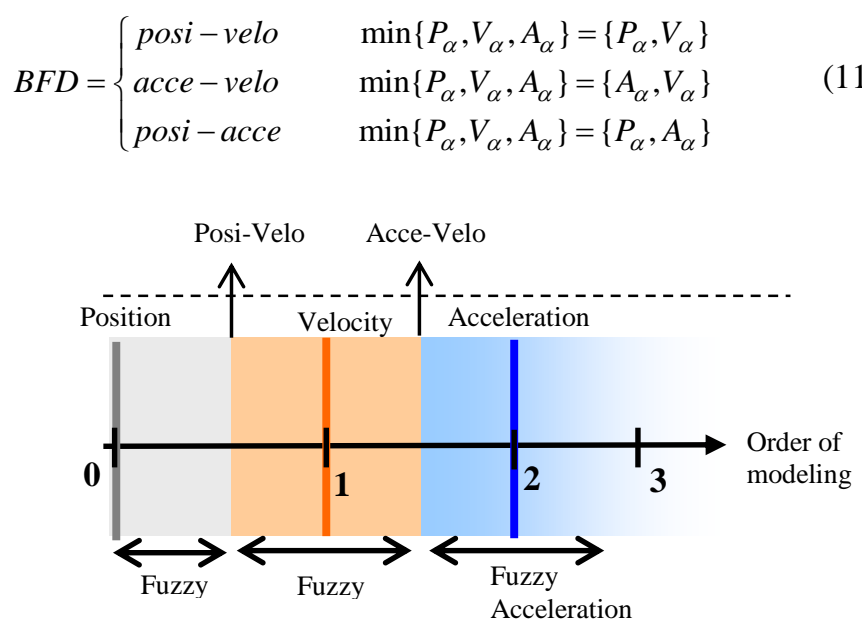

Fig.1. Fuzzy modeling domains of FO motion equations.

Eq.(11) gives arithmetical definitions of posi-velo, acce-velo and posi-acce domains seen in Fig. 1

\section{APPLICATION ON MOTION ANALYSIS}

In this section, we give a discussion on the motion model defined by a second-order polynomial position equation. This example model can be expressed as,

$$
y=\sum_{i=0}^{2} a_{i} t^{i}
$$

FOD model space can be obtained according to equation (2) as follows.

$$
\psi^{\alpha}=D^{\alpha} y=\sum_{i=0}^{2} a_{i} \frac{\Gamma(i+1)}{\Gamma(i-\alpha+1)} t^{i-\alpha}
$$

Fig.2 shows sample models from fuzzy position domain in (a), from fuzzy velocity domain in (b) and from fuzzy acceleration domain in (c) for a second-degree polynomial position model with $a_{\mathrm{i}}=1$ and $n=2$. It was observed from Fig. 2 that FOD models in the same fuzzy domain are more similar in characteristics than models of other domains (fuzzy similarity). As the order of FOD model approaches to the IOD model in its domain, it becomes more similar in characteristic. Thus, for a given FOD model characteristic, one can roughly classify it to a fuzzy domain by visual inspection of characteristics. For instance, derivative model of $\alpha=0.9$ in Fig.3(b) can classify to fuzzy velocity domain, visually. Fig.3 illustrates fuzzy distance 
mappings containing distances to position $\left(P_{\alpha}\right)$, distances to velocity $\left(V_{\alpha}\right)$ and distances to acceleration $\left(A_{\alpha}\right)$. Boundaries of these fuzzy modeling domains are indicated by vertical dash lines, where the distances to concept models are equal. As seen in the Fig.3, boundaries of fuzzy domains, named as Posi-Velo for $P_{\alpha}=V_{\alpha}$ and Acce-Velo for $V_{\alpha}=A_{\alpha}$, are not at $\alpha=0.5$ and $\alpha=1.5$ respectively. This confirms that fuzzy domains do not have to share FOD modeling set equally. Changing the second order polynomial coefficients does not apparently change the location of fuzzy domain boundaries. But, it can considerably alter distance magnitudes of the domain boundaries. Fig.4 shows this effect for various member of the polynomial family. These results illustrate us that fuzzy domains of fractional ordermodeling space are quite stable in $f_{n}$ polynomial family (Fuzzy domain stability).

\section{CONCLUSIONS}

We presented an analysis to reveal similarity of fractionalorder models of physical systems and try to gain prospect for physical interpretation of FOD models. For this proposes, continuous modeling space of FOD modeling is divided fuzzy domains of IOD models by using an absolute differences metrics. We present an example discussion on a second-order polynomial motion equations, which can model motion of system with a constant acceleration. Findings of this application example can be generalized for the mechanic system models involving a velocity and acceleration parameters. For instance, chemical reaction velocity models, population growing velocity models. This study may contribute to better evaluation of FOD system models and helps physical interpretation of these models.
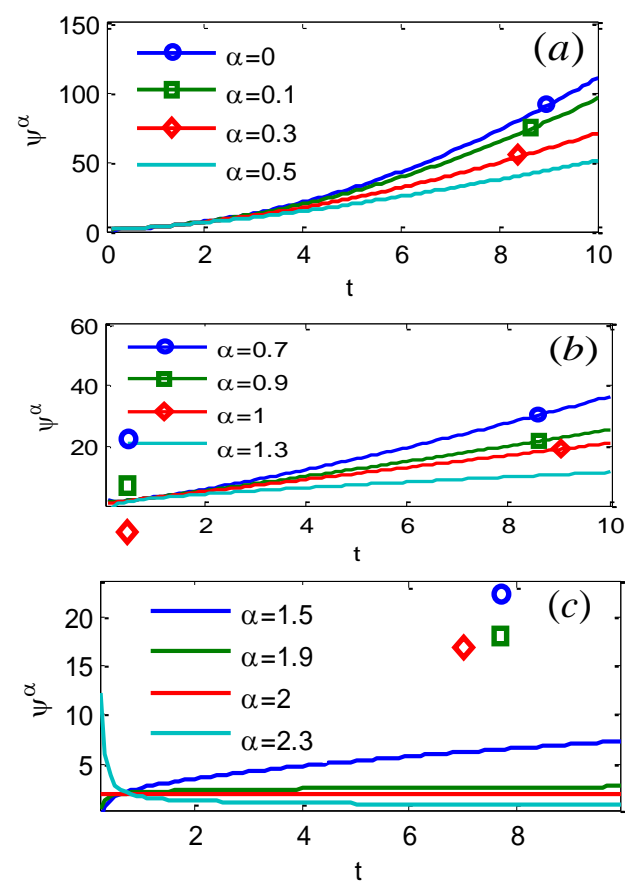

Fig.2. (a) Fuzzy position model examples of motion equations. (b) Fuzzy velocity model examples of motion equations. (c) Fuzzy acceleration model examples of motion equations.

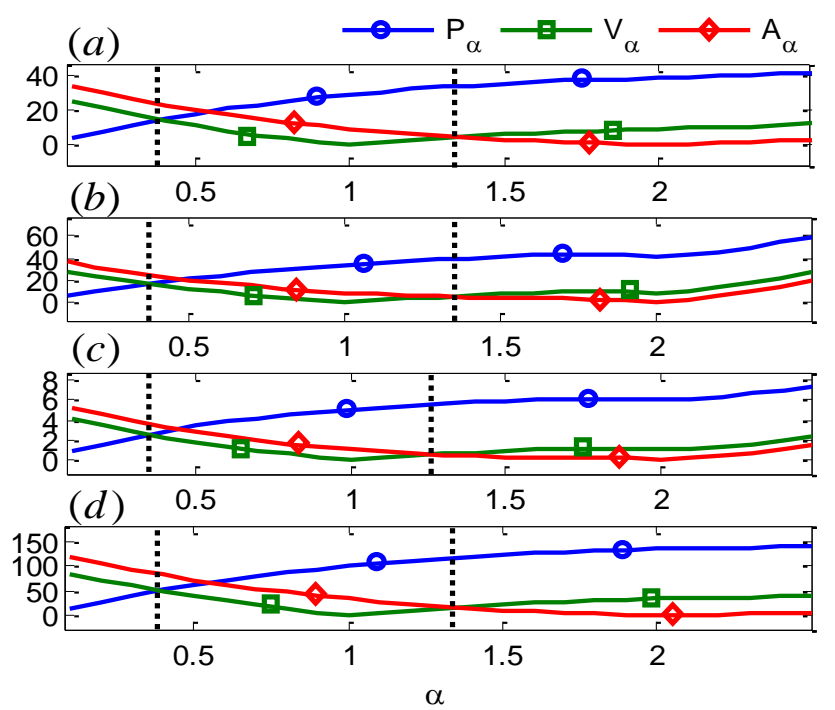

Fig.3. Fuzzy distance mapping to conceptualized IOD models which are $P_{\alpha}, V_{\alpha}$ and $A_{\alpha}$ for the motion equation modeling with $a_{i}=1$ and $t=10$.

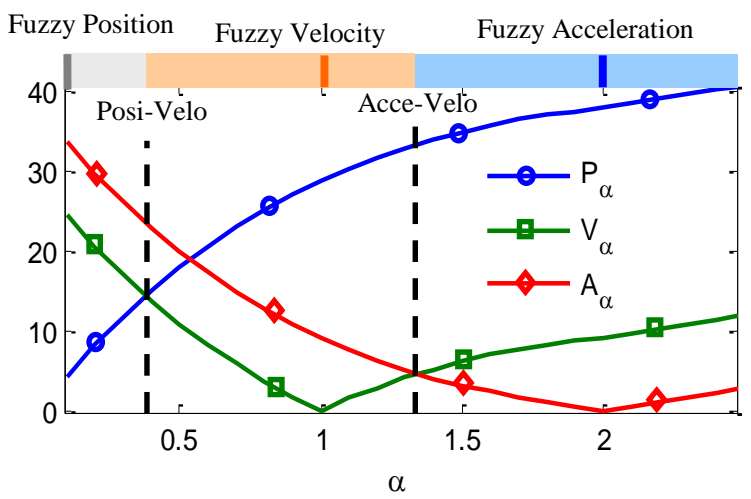

Fig.4. Comparison of distance mapping of fuzzy domains for various secondorder polynomials.

$a_{2}=1, a_{1}=1, a_{0}=1$ in (a), $a_{2}=1, a_{1}=0, a_{0}=1$ in (b), $a_{2}=0, a_{1}=1$, $a_{0}=1$ in (c),

$a_{2}=4, a_{1}=1, a_{0}=0$ in (d) for $t=10$. Dash lines indicate boundaries of fuzzy domains.

\section{REFERENCES}

[1] I. Podlubny, Fractional Differential Equations Vol. 198, Mathematics in Science and Engineering, Academic Press, New York and Tokyo, 1999.

[2] K. B. Oldham and J. Spanier, The Fractional Calculus: Theory and Applications of Differentiation and Integration to Arbitrary Order, Dover Books on Mathematics, 2006.

[3] B. Ross, Fractional Calculus and its Applications, Springer - Verlag, Berlin, 1975.

[4] J. Sabatier, O. P. Agrawal and J. A. Machado, Advances in Fractional Calculus: Theoretical Developments and Applications in Physics and Engineering Springer, 2007.

[5] A. A. Kilbas, H. M. Srivastava and J. J. Trujillo, Theory and Applications of Fractional Differential Equations, Elsevier, 2006.

[6] S. Das, Functional Fractional Calculus for System Identification and Controls, Springer, 2007.

[7] T. M. Saleh, M. Haeri, Rational approximations in the simulation and implementation of fractional-order dynamics: A descriptor system approach, Automatica,vol.46, no.1, pp.94-100, 2010. 
[8] A. E. Matouk, Chaos, feedback control and synchronization of a fractional-order modified autonomous van der Pol-Duffing circuit, Communications in Nonlinear Science and Numerical Simulation, 16, 2 pp.975-986, 2011.

[9] A. S. Elwakil, Fractional-order circuits and systems: An emerging interdisciplinary research area, Circuits and Systems Magazine, IEEE, vol. 10, no.4, pp.40-50, 2010

[10] S. Lu, F.J. Molz, G.J. Fix, Possible problems of scale dependency in applications of the three-dimensional fractional advection-dispersion equation to natural porous media, Water Resources Research, vol.38, pp. $41-47,2002$.

[11] L. Kexue, P. Jigen, Fractional resolvents and fractional evolution equations, Applied Mathematics Letters, vol.25, pp.808-812, 2012.

[12] A.M.A. El-Sayed, A.E.M. El-Mesiry, H.A.A. El-Saka, On the fractionalorder logistic equation, Applied Mathematics Letters, vol.20, pp.817-823, 2007

[13] E.M. El-Mesiry, A.M.A. El-Sayed, H.A.A. El-Saka, Numerical methods for multi-term fractional (arbitrary) orders differential equations Appl. Math. Comput. vol.160, pp.683-699, 2005.

[14] R.E. Gutiérrez, J.M. Rosário and J.A.T. Machado, Fractional Order Calculus: Basic Concepts and Engineering Applications, Hindawi Publishing Corporation Mathematical Problems in Engineering, vol.19, 2010.

[15] G.W. Leibnitz. Letter from hanover, germany, september 30, 1695 to g. a l'hospital. Leibnizen Mathematische Schriften. Olms Verlag Hildesheim, Germany, 1962, First published in 1849.

[16] J. A. T. Machado, A probabilistic interpretation of the fractional-order differentiation Fractional Calculus and applied Analysis, vol. 6 pp.73-80, 2003.

[17] I. Podlubny, Geometric and physical interpretation of fractional integration and fractional differentiation, Fractional Calculus \& Applied Analysis, vol. 5 pp.367-386, 2002.

[18] M. Moshrefi-Torbati, J.K. Hammond, Physical and geometrical interpretation of fractional operators,Journal of the Franklin Institute, vol. 335, pp.1077-1086, 1998.

[19] F.J. Molz, G.J. Fix, S. Lu, A physical interpretation for the fractional derivative in Levy diffusion, Applied Mathematics Letters, vol.15, pp. 907-911, 2002

[20] C. Giannantoni, The problem of the initial conditions and their physical meaning in linear differential equations of fractional order, Applied Mathematics and Computation, vol. 141, pp. 87-102, 2003.

[21] M. Dorota, D. F. M. Torres, Modified optimal energy and initial memory of fractional continuous-time linear systems, Signal Processing, vol.91, no.3, pp.379-385, 2011.

[22] R. E. Gutiérrez, J. M. Rosário, J. T. Machado, Fractional order calculus: basic concepts and engineering applications, Mathematical Problems in Engineering, vol.2010, 2010.

\section{BIOGRAPHIES}

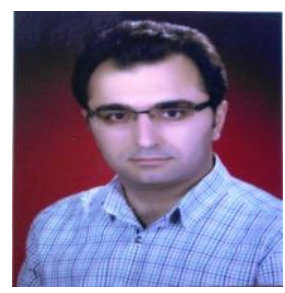

ABDULLAH ATES received his B. Sc. Degree in Electrical and Electronics Engineering from Erciyes University in 2011. He received his master degree in Computer Engineering from Inonu University in 2013. His research interests include optimization algorithm, analytical and numerical optimization method, fractional order control systems, fractional order calculus and its application, linear and nonlinear optimization algorithm flight control.

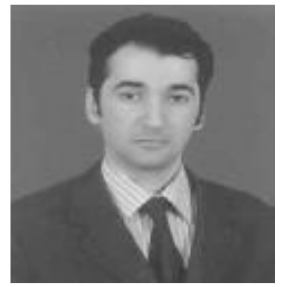

BARIS BAYKANT ALAGOZ was graduated from University of Istanbul Technical University department of Electronics and Communication Engineering in 1998. He has PhD degree from Inonu University, department of Electrical-Electronics Engineering in 2015.

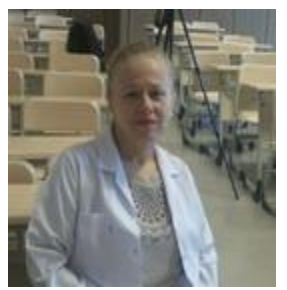

G.T. ALISOY was graduated with honors from the Faculty of Mathematics and Mechanics of the Azerbaijan State University. Her M.Sc.(85') and Ph.D. (97').She received Assoc. Prof. degree in Analysis and Theory of functions from High Education Council of Turkey in 2012. Currently, she is Assos.Professor, in Mathematical Department of Namik Kemal University. Her research interests are, integral representation of multi-package variable functions, differential properties of functions, mathematical modeling of engineering problems

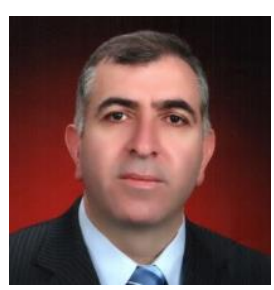

CELALEDDIN YEROĞLU received his B.Sc. degree in Electrical and Electronics Engineering from Hacettepe University in 1990. He received his first Ph.D. degree in Computer Engineering from Trakya University in 2000 and his second Ph.D. degree in Electrical and Electronics Engineering from Inonu University in 2011. His research interests include fractional order control systems, robust control, nonlinear control, modeling and simulation, optimization algorithm, flight control.

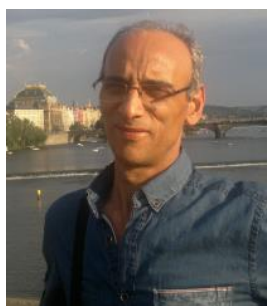

HAFIZ Z. ALISOY was graduated from Moscow Technical University department of Electro-Physics Engineering 1982. He had his PhD degree from USSR Science Academy Physics Institute of P.N. Lebedyev and Doctor of Sciences degree (DSc) from International Ecology-Energy Academy. He became as Full Professor in 1995. He received award of young Scientist. He works at Namik Kemal University, Department of Electronics and Communication Engineering. 